\title{
Genetic diversity of genes involved in fatty acid biosynthesis in a collection of flax cultivars
}

\author{
Kezimana P. ${ }^{1,2 *}$, Rozhmina T.A. ${ }^{1,3}$, Krasnov G.S. ${ }^{1}$, Novakovskiy R.O. ${ }^{1}$, \\ Povkhova L.V. ${ }^{1,4}$, Pushkova E.N. ${ }^{1}$, Romanova E.V. ${ }^{2}$, Dmitriev A.A. ${ }^{1}$, Melnikova N.V. ${ }^{1}$ \\ ${ }^{1}$ Engelhardt Institute of Molecular Biology, RAS, Moscow, Russia \\ ${ }^{2}$ Peoples' Friendship University of Russia (RUDN University), Moscow, Russia \\ ${ }^{3}$ Federal Research Center for Bast Fiber Crops, Torzhok, Russia \\ ${ }^{4}$ Moscow Institute of Physics and Technology, Dolgoprudny, Russia \\ *e-mail:k1par@mail.ru
}

Flax (Linum usitatissimum L.) is one of the major sources of omega-3 fatty acids (FAs), which provide health benefits for humans. Flaxseed oil is composed of palmitic (PAL, C16:0), stearic (STE, C18:0), oleic (OLE, C18:1), linoleic (LIO, C18:2), and linolenic (LIN or ALA, C18:3) acids, with high levels of LIN and moderate levels of LIO, the essential FAs, being attributed the nutraceutical properties of flaxseed. Genetic control of FA biosynthesis in flax has been studied and genes encoding the enzymes that perform FA synthesis have been identified, however, there is still little information regarding the relationship between the genetic diversity of these genes and fatty acid composition in flax. In the present study, our goal was to analyze the genetic variability for $S A D$ (stearoyl-ACP desaturase) and FAD (fatty acid desaturase) genes in flax by sequencing these genes in 288 flax accessions with different proportion of FAs, obtained from the Institute for Flax (Torzhok, Russia). For genetic variation analysis, we used generated DNA sequences with an average coverage of 100x for an individual sample from the Illumina platform. Genetic variation data were correlated with FA composition data, in order to determinate the key polymorphisms leading to different proportion of FAs that will provide further information in order to understand the genetic factors controlling FA composition in flax.

Acknowledgements: This work was performed within the framework of the Program of fundamental research for state academies for 2013-2020 years (No. 01201363824) and was funded by RFBR according to the research project No. 17-29-08036. 